\title{
Was wünschen sich die Allergologen?
}

$\mathrm{N}$ otfallseminare stehen bei allergologisch tätigen Ärzten ganz oben auf der Wunschliste der Trainingsangebote für ihr Team und sich selbst. Das hat eine Befragung von Novartis Pharma ergeben, an der 1.598 deutsche Ärzte beteiligt waren (Abb.). Untersucht wurden die drei Bereiche „Ärztliche Fortbildung und CME“, „Schulung und Training für Ihr Praxisteam“ sowie „Weitere Serviceangebote". Mit 553 Teilnehmern war die Fachgruppe der Dermatologen am stärksten vertreten, gefolgt von Pädiatern (444), Hals-Nasen-Ohrenärzten (352) und Pneumologen (124).

Das Thema Notfallmanagement lag bei allen Fachrichtungen ganz weit vorn - sowohl bei den Ärzten selbst (Platz 2) als auch in der Kategorie „Schulung und Training für Ihr Praxisteam“" (Platz 1). Mehr Unterstützung für ihr Team könnten sich Allergologen außerdem auf den Gebieten „Praxisorganisation“ und „Patientenkommunikation“ vorstellen. Und wo wünschten sich die Befragten noch zusätzlich Unterstützung? Besonders nachgefragt wurden Services wie „Medizinischer Praxisbedarf“, „Patienteninformationsmaterial“ und „Literaturservice zur Allergologie“.
„Ziel der Umfrage war, das Serviceangebot von Novartis noch besser an die Wünsche der allergologisch tätigen Ärzte anzupassen. Dies folgt dem Anspruch unseres Unternehmens, ein zuverlässiger Partner für Fachärzte und Patienten in der spezifischen Immuntherapie zu sein", so Kim Abbenhaus, Leiterin Allergie und Atemwege bei Novartis Pharma. Schon jetzt bietet Novartis mit dem Allergologie Spezialisten Service ein umfangreiches Servicepaket. Offeriert werden neben dem Notfalltraining auch diverse Kommunikationsseminare und medizinischgesundheitspolitische Abende, bei denen neue wissenschaftliche Erkenntnisse ebenso auf der Agenda stehen wie Abrechnungsstrategien oder Gesundheitspolitik. Eine Vielzahl an nützlichen Unterlagen wie Patientenbroschüren, Praxisposter, Einverständniserklärungen oder ein Pollen- und Kreuzallergenkalender können darüber hinaus rund um die Uhr unter der Webadresse www.depigoid.de bestellt werden.

Nach Informationen von Novartis Pharma, Nürnberg

\section{Pråparate auf einen Dreh}

Die Immuntherapiepräparate von Allergopharma erfüllen schon jetzt in hohem Maße die Forderungen der Therapieallergene-Verordnung: Insgesamt 45 davon sind bereits zugelassen, für zahlreiche weitere Extrakte ist die Zulassung in Vorbereitung. Um angesichts dieses großen Produktportfolios den Überblick behalten zu können, hat das Unternehmen für Ärzte eine neuartige Drehscheibe entwickelt: Nach Einstellen der für die Beschwerden des jeweiligen Patienten relevanten Allergene lässt sich auf einen Blick ablesen, welches der Präparate von Allergopharma für eine spezi-

fische Immuntherapie in Frage kommt. Ebenso wird der Zulassungsstatus des jeweiligen Präparates angezeigt. Auf der Rückseite der Scheibe ist zusätzlich ein ebenfalls drehbarer Pollenflugkalender integriert.

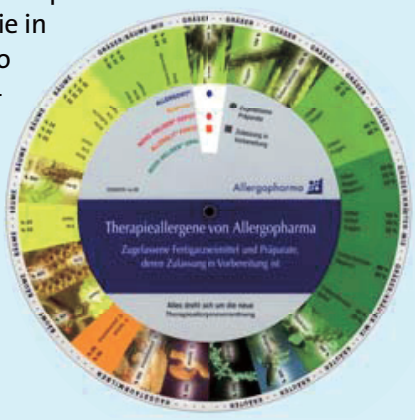

Die Info-Drehscheibe kön-

nen Allergologen kostenlos entweder über den Allergopharma-Außendienst oder direkt bei Allergopharma, Tel. (o 40) $72765-4$ oo oder Fax -2 68 bestellen.

Nach Informationen von Allergopharma, Reinbek

\section{Daten auf einen Klick}

In dem Unternehmen ALK-Abelló wird derzeit ein neues Clinical Trial Management System etabliert. Ein solches System wird unterstützend bei der Planung, Vorbereitung, Durchführung und Auswertung von klinischen Studien eingesetzt. In den vergangenen Monaten wurde in Zusammenarbeit mit dem Unternehmen BSI eine auf die Bedürfnisse von ALK-Abelló zugeschnittene Version erstellt, die eine optimale Dokumentation aller relevanten Studienprozesse gewährleistet. Weltweit werden ca. 100 Forschungsmitarbeiter mit der neuen Software arbeiten. Alle notwenigen Formulare, Reports und Daten werden in diesem System erfasst. Daraus resultiert ein umfassender Überblick über laufende und geplante Studien sowie über die Ergebnisse von bereits absolvierten Studien.

Nach Informationen von ALK-Abelló, Wedel 